\title{
Strengere
}

\section{Verwendungskriterien für Appetitzügler}

\author{
A. Schneider, S. Borner
}

Die Interkantonale Kontrollstelle für Heilmittel (IKS) hat im März 2000 eine Überprüfung der zentral wirkenden appetithemmenden Medikamente (Anorektika) abgeschlossen.

Konkret ausgelöst wurde die Überprüfung durch das Bekanntwerden von Herzklappenschäden bei Frauen, die in den USA Fenfluramin und teilweise gleichzeitig auch andere Anorektika eingenommen hatten, insbesondere Phentermin (sogenannte Fen/ Phen-Kombination) [1]. Diese Vorfälle führten im September 1997 zum sofortigen Rückzug aller Fenfluramin- und Dexfenfluraminhaltigen Präparate [2]. Als Konsequenz wurden anschliessend auch die übrigen Anorektika überprüft.

\section{Ziele und Ergebnisse der Überprüfung}

In erster Linie wurde beurteilt, welche Risiken mit einer Einnahme von Anorektika einhergehen und mit welchen Massnahmen die Sicherheit ihrer Anwendung verbessert werden kann.

Die IKS kam zum Schluss, dass der kurzzeitige Einsatz (4-8 Wochen*) von Appetitzüglern bei Patientinnen und Patienten mit deutlichem ernährungsbedingtem Übergewicht (Body Mass Index mindestens 30) im Rahmen eines umfassenden Behandlungskonzeptes üblicherweise keine unzulässigen Risiken birgt. Allerdings sind dabei verschiedenste Anwendungseinschränkungen und Vorsichtsmassnahmen zu beachten (siehe Kasten).

Um die Sicherheit bei der Verschreibung und der Medikamentenabgabe $\mathrm{zu}$ verbessern, wurden die Fach- und Patienteninformationen der Präparate im Hinblick auf Risiken und Vorsichtsmassnahmen

\footnotetext{
* Zu Sibutramin liegen Sicherheits- und Wirksamkeitsdaten für die Anwendung bis zu zwei Jahren vor, weshalb bei diesem Präparat auch in Zukunft längere unterstützende Therapien möglich sind.
}

Korrespondenz:

Dr. Stefan Borner

IKS

Erlachstrasse 8

CH-3000 Bern 9

Tel. 0313220384

E-mail stefan.borner@iks.admin.ch

\section{Auszug aus dem Kompendiumstext}

Indikation

Zur unterstützenden Behandlung von ernährungsbedingtem Übergewicht bei Patienten mit einem BMI von mind. $30 \mathrm{~kg} / \mathrm{m}^{2}$, die auf geeignete gewichtsreduzierende Massnahmen allein ungenügend angesprochen haben.

Das Präparat soll nur kurzfristig (4-8 Wochen*) angewendet werden.

Von einer wiederholten Anwendung wird abgeraten.

Die Behandlung soll im Rahmen eines umfassenden Konzeptes erfolgen, das diätetische, medizinische und evtl. psychotherapeutische Methoden einschliesst.

\section{Wichtige Kontraindikationen}

Zerebro- oder kardiovaskuläre Erkrankungen, psychische Erkrankungen, Hyperthyreose, Phäochromozytom, Glaukom, Prostatahyperplasie.

Keine gleichzeitige Behandlung mit einem anderen zentral wirkenden Appetitzügler (erhöhtes Risiko für pulmonale arterielle Hypertonie und Herzklappenschäden).

Vorsichtsmassnahmen

Belastungsdyspnoe lässt auf die Möglichkeit einer pulmonalen arteriellen Hypertonie schliessen. Untersuchung durch Facharzt notwendig.

Das Reaktionsvermögen kann verändert werden (Strassenverkehr oder Bedienen von Maschinen).

\section{Interaktionen (Auszug)}

Die Kombination mit anderen ZNS-wirksamen Medikamenten (insbesondere Sympathomimetika und MAO-Hemmern) sowie oralen Antidiabetika muss vermieden werden. Eine Interaktion mit MAOHemmern ist noch während 14 Tagen nach deren Absetzung möglich.

$\mathrm{Zu}$ beachten ist, dass auch manche Husten-, Asthma-, Schnupfen- und Erkältungsmittel sympathomimetische Wirkstoffe enthalten.

aktualisiert und vereinheitlicht. Überdies sind alle Anorektika rezeptpflichtig, und eine Packung darf maximal für eine 30tägige Therapiedauer ausreichen.

Relevante Nebenwirkungen, die auftreten können, lassen sich zumeist auf den zentralen oder peripheren adrenergen und/oder serotoninergen Wirkmechanismus dieser Präparate zurückführen.

Mögliche schwerwiegende Nebenwirkungen der Anorektika beinhalten eine Abhängigkeit, eine arterielle Hypertonie und die Entwicklung einer pulmonal-arteriellen Hypertonie (PAH).

Die PAH kann bei etwa 50\% der Betroffenen zum Tod führen. Das Risiko für eine PAH ist zwar niedrig, steigt aber bei einer Behandlungsdauer von 3 Monaten und mehr stark an [3]. 
Es ist bekannt, dass die Anorektika allgemein eine beschränkte Wirksamkeit bezüglich Gewichtsverlust und Halten des erreichten Gewichtes haben. Es kann je nach Substanz und Behandlungskonzept von einer zusätzlichen Reduktion um 5\% bis maximal 10\% des Ausgangsgewichtes ausgegangen werden.

Bisher liegen noch zu keinem Präparat Untersuchungen vor, die zeigen, dass mit dem Gewichtsverlust auch die Morbidität oder Mortalität der behandelten Patienten günstig beeinflusst werden.

\section{Wie sieht das internationale Umfeld aus?}

Im März dieses Jahres erfolgte in der Europäischen Union ein Erlass, welcher inskünftig in den EULändern das Inverkehrbringen praktisch sämtlicher zentral wirksamer Anorektika verbietet.

Begründet wurde dieser Schritt vorwiegend damit, dass bei den betroffenen, teilweise seit vielen Jahren erhältlichen Medikamenten der Langzeitnutzen ungenügend nachgewiesen sei*. Verschiedene EU-Vertriebsfirmen haben deswegen den europäischen Gerichtshof angerufen.

In den USA wurden nach dem Rückzug von Fenfluramin und Dexfenfluramin im Jahre 1997 keine weiteren Massnahmen getroffen.

\section{Weitere Schritte in der Schweiz}

Die aktualisierten Fach- und Patienteninformationen werden im Supplementum 3 des Arzneimittelkompendiums 2000 publiziert. Ab Juni 2000 dürfen zudem nur noch Medikamentenpackungen ausgeliefert werden, die die neue Patienteninformation enthalten und die für maximal 30 Tage reichen.

Sollte sich zeigen, dass trotz der getroffenen Massnahmen Nutzen und Risiken einer Anwendung zentral wirksamer Anorektika in einem ungünstigen Verhältnis stehen, wird die IKS eine erneute Überprüfung dieser Präparategruppe vornehmen.

\section{Literatur}

1 Connolly HM, Crary JL, McGoon MD, Hensrud DD, Edwards BS, Edwards WD, Schaff HV. Valvular heart disease associated with fenfluramine-phentermine. N Engl J Med 1997;337(9):581-8.

2 Interkantonale Kontrollstelle für Heilmittel IKS. Herzklappenerkrankungen bei Anorektika-Kombinationstherapie mit Phentermin und Fenfluramin. Schweiz Ärztezeitung 1997: 78(35):1264.

3 Abenhaim L, Moride Y, Brenot F, Rich S, Benichou J, Kurz X, et al. Appetite-suppressant drugs and the risk of primary pulmonary hypertension. International Primary Pulmonary Hypertension Study Group. N Engl J Med 1996;335(9):609-16. 